\title{
Sentinel lymph node detection using computed tomography lymphography is accurate after neoadjuvant chemotherapy for breast cancer
}

\author{
HIRONOBU UE ${ }^{1}$, YASUHIRO OGAWA ${ }^{1}$, SHINJI KARIYA ${ }^{1}$, KIMIKO NAKATANI ${ }^{1}$, KAZUHIRO TSUZUKI $^{1}$, \\ KANA MIYATAKE $^{1}$, HIRONORI MAEDA $^{2}$, YOSUKE TANAKA ${ }^{2}$ and SHIRO SASAGURI ${ }^{2}$ \\ Departments of ${ }^{1}$ Diagnostic Radiology and Radiation Oncology, and ${ }^{2}$ Surgery II, Kochi \\ University Hospital, Medical School, Kochi University, Kochi 783-8505, Japan
}

Received November 29, 2006; Accepted January 15, 2007

\begin{abstract}
For breast cancer patients who have undergone neoadjuvant chemotherapy (NAC), a sentinel lymph node biopsy (SLNB) has not been recommended until recently. This is due to the possible lymph-flow interruption caused by fibrotic changes following chemotherapy and possible increases in false-negative cases. We investigated the changes in the lymph-flow and the detection of sentinel lymph nodes (SLNs) using computed tomography (CT) lymphography before and after NAC. We enrolled 53 patients with breast cancer who had undergone CT lymphography between May 2004 and April 2006. In total, 75 examinations were performed; 44 before NAC and 31 just after NAC. The CT lymphography procedures were approved by the medical ethics committee of our university. After a comprehensive explanation, written informed consent was obtained from all the patients, prior to enrollment in the study. Differences in changes in the lymph-flow, detection of SLNs, and changes in the number of detected SLNs were examined before and after NAC. Differences in the categoric variables were analyzed using the Chi-square test or Fisher's exact test. The identification rate $(90.5 \%)$ of the SLNs following NAC was higher than the one before NAC $(79.5 \%)$. However, no statistically significant difference was noted. No interruptions to the lymph-flow prevented the detection of SLNs by NAC. By performing CT lymphography before and after NAC, the interruption to the lymph-flow can be checked and the site of SLNs can be identified prior to surgery. We found that SLNB is recommended for breast cancer patients with or without NAC.
\end{abstract}

Correspondence to: Dr Hironobu Ue, Department of Diagnostic Radiology and Radiation Oncology, Medical School, Kochi University, Oko-cho, Nankoku-shi, Kochi 783-8505, Japan

E-mail: jm-ueh@kochi-u.ac.jp

Key words: sentinel lymph node, neoadjuvant chemotherapy, computed tomography lymphography, breast cancer

\section{Introduction}

Surgical procedures for patients with breast cancer are becoming less invasive, and breast-conservation treatment (BCT) is now extensively performed. Additionally, due to the increase in the number of patients whose primary lesion and/or axillary lesion are totally controlled by neoadjuvant chemotherapy (NAC), the number of possible candidates for $\mathrm{BCT}$ is also increasing.

Previously, axillary lymph node dissection (ALND), which involves total resection of the regional lymph nodes, was considered to be essential for breast surgery for patients with breast cancer, and was performed worldwide. It has been observed however, that ALND decreases the rate of axillary recurrence without any evidence of survival benefits for patients with breast cancer (1). In addition, axillary irradiation decreases the rate of axillary recurrence for breast cancer patients with clinically-negative axillary lymph nodes $(2,3)$. Therefore, ALND is becoming a method for recognizing the existence or absence of lymph node metastases, and/or preventing axillary failure, and not for obtaining survival benefits. Due to the reasons mentioned above, the number of patients undergoing sentinel lymph node biopsy (SLNB) instead of ALND is rapidly increasing. As SLNB can contribute to a significant decrease in post-operative complications (4), this method should be more widely recommended in the future.

Recently, certain reports have been published on the clinical results of SLNB after NAC (5-7). For patients with axillary lymph node metastases, SLNB performed after NAC can provide a measure of the therapeutic response of these lymph node metastases to chemotherapy. Thus, patients whose metastatic lymph nodes are free of cancer cells after NAC, could be spared from ALND. Furthermore, the degree of residual disease in the axillary nodes after NAC has a powerful independent prognostic value (8). However, SLNB after NAC is still in the clinical testing stage. For patients who undergo NAC, SLNB has not been recommended until now, due to the possible interruption to the lymph-flow caused by fibrotic changes following chemotherapy $(9,10)$ and possible increases in false-negative cases $(7,11)$. Therefore, for patients who undergo NAC, SLNB has been performed 
Table I. Characteristics of patients who underwent CT lymphography.

\begin{tabular}{llll}
\hline & Before NAC & After NAC & \\
& 44 cases & 31 cases & \\
\hline Tumor size (mm) & $26.1 \pm 16.2$ & $22.5 \pm 12.6$ & mean \pm SD \\
$\leq 20 \mathrm{~mm}$ (cases) & 23 & 18 & \\
$>20 \mathrm{~mm}$ (cases) & 21 & 13 & \\
Nodal status & & & \\
$\quad$ Negative (cases) & 35 & 26 & \\
Positive (cases) & 9 & 5 & \\
Age (years) & $54.2 \pm 11.3$ & $53.2 \pm 10.2$ & mean \pm SD \\
Body mass index & $22.3 \pm 3.2$ & $23.2 \pm 3.3$ & mean \pm SD \\
Region & & & \\
Inner (A,B) (cases) & 12 & 10 & \\
Outer (C,D) (cases) & 32 & 21 & \\
\hline
\end{tabular}

CT, computed tomography; NAC, neoadjuvant chemotherapy.

prior to the initiation of NAC (12-14). The procedure has otherwise not been employed, in spite of the recent trend toward less-extensive breast surgery.

However, it can be previously confirmed if the lymphflow changes before and after performing NAC for patients with breast cancer. In the present study, we investigated the changes in the lymph-flow and the detection of sentinel lymph nodes (SLNs) using computed tomography (CT) lymphography (15-17) before and after NAC.

\section{Materials and methods}

Patients. We enrolled 53 patients with breast cancer who had undergone CT lymphography between May 2004 and April 2006. In total, 75 examinations were performed (Table I); 44 of the 75 examinations were performed before NAC (and/or for patients without chemotherapy) while the other 31 examinations were performed just after NAC. The procedures for CT lymphography were approved by the medical ethics committee of our university. After a comprehensive explanation, written informed consent was obtained from all the patients, prior to enrollment in the study.

NAC performed pre-operatively consisted of three to four courses of CAF chemotherapy (each chemotherapy administration consisted of $600 \mathrm{mg} / \mathrm{m}^{2}$ cyclophosphamide, $600 \mathrm{mg} / \mathrm{m}^{2}$ 5-fluorouracil and 20-40 mg pirarubicin, every two weeks) and anti-estrogen agent (tamoxifen citrate for pre-menopausal women and toremifene citrate for postmenopausal women) according to our treatment strategy for patients with breast cancer (18).

CT lymphography. CT lymphography was performed in the supine position using an Aquillion 16-detector row CT scanner (Toshiba Medical Systems, Tochigi, Japan), keeping the bilateral arms on the top of the head and the bilateral upper extremities vertical, resembling the surgical position.

Image collection $(120 \mathrm{kV}, 50 \mathrm{mAs}, 0.5 \mathrm{sec}$ scan time) was started at $1 \mathrm{~min}$ and $3 \mathrm{~min}$ after the injection of $2 \mathrm{ml}$ iodine contrast medium (Iopamidol, Schering, Germany) subdermally just above the primary tumor and just beneath the areola, respectively. Following the injection of the contrast medium, a massage was performed for $30 \mathrm{sec}$ at each injected region. To relieve pain due to the contrast medium injection, Penles (Lidocaine sheet, Wyeth, USA.) had been attached to the regions $3 \mathrm{~h}$ before the examination. The lymph-flow and SLNs were detected on thin slice axial images (1-mm slice thickness) and 3D-CT images (Fig. 1). The images were interpreted prospectively and evaluated independently by 2 radiologists, each of whom had $>5$ years radiological experience, including experience with breast imaging. Differences in assessment were resolved by a consensus. Both observers were informed that the patients had breast cancer. However, they were completely unaware of the existence of NAC, pathological staging of cancer, patient age and body mass index (BMI).

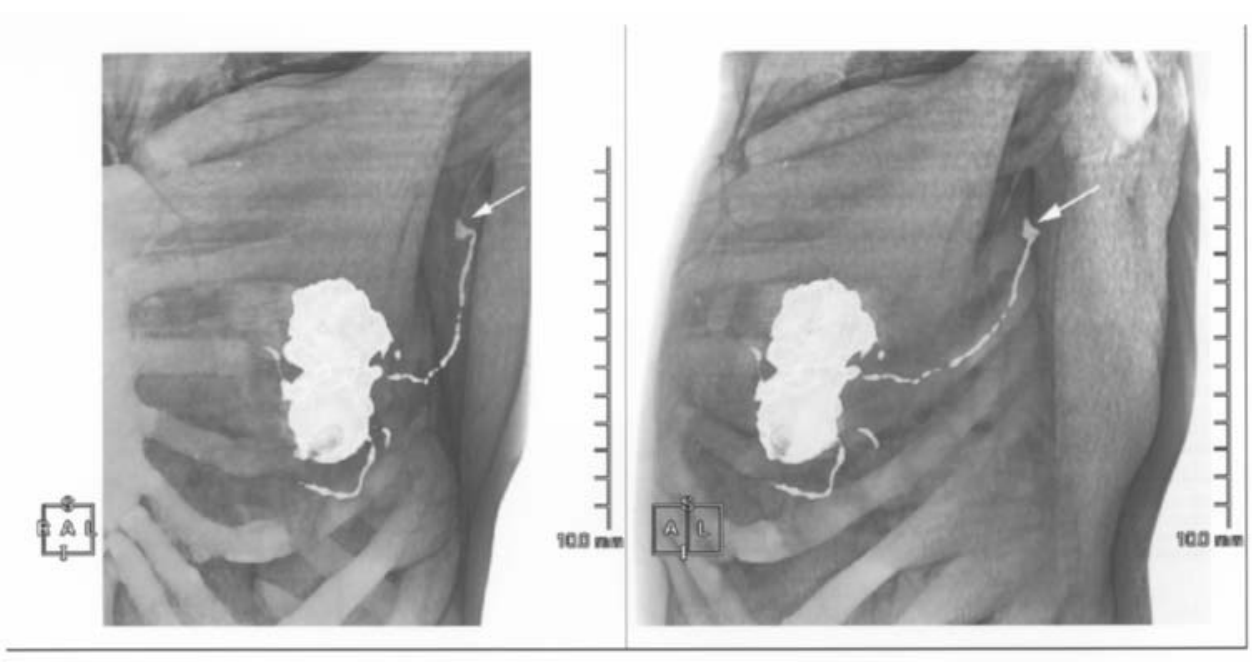

Figure 1. 3D-computed tomography (CT) lymphography. The lymph flow from the subareolar and peritumoral injection sites drains into the axillary lymph node $(\rightarrow)$. The lymph node into which the contrast media flows first is regarded as the sentinel lymph node. 3D-CT lymphography can confirm the anatomy of this lymph flow. 
Table II. Identification rate of sentinel lymph nodes.

\begin{tabular}{|c|c|c|c|c|c|}
\hline & No. attempted & Detected cases & Undetected cases & Identification rate $(\%)$ & P-value \\
\hline Overall & 75 & 63 & 12 & 84 & \\
\hline NAC & & & & & $0.176^{\mathrm{a}}$ \\
\hline Before & 44 & 35 & 9 & 79.5 & \\
\hline After & 31 & 28 & 3 & 90.3 & \\
\hline Tumor size & & & & & $0.024^{\mathrm{b}}$ \\
\hline$\leq 2 \mathrm{~cm}$ & 41 & 38 & 3 & 92.7 & \\
\hline$>2 \mathrm{~cm}$ & 34 & 25 & 9 & 73.5 & \\
\hline Nodal status & & & & & $<0.01^{\mathrm{a}}$ \\
\hline Negative & 61 & 57 & 4 & 93.4 & \\
\hline Positive & 14 & 6 & 8 & 42.9 & \\
\hline Age (years) & & & & & $0.100^{\mathrm{b}}$ \\
\hline$<55$ & 40 & 31 & 9 & 77.5 & \\
\hline$\geq 55$ & 35 & 32 & 3 & 91.4 & \\
\hline BMI & & & & & $0.647^{\mathrm{b}}$ \\
\hline$<23$ & 42 & 36 & 6 & 85.7 & \\
\hline$\geq 23$ & 33 & 27 & 6 & 81.8 & \\
\hline Region & & & & & $0.756^{\mathrm{a}}$ \\
\hline Inner & 22 & 18 & 4 & 81.8 & \\
\hline Outer & 53 & 45 & 8 & 84.9 & \\
\hline Period & & & & & $0.638^{\mathrm{a}}$ \\
\hline 2004 & 31 & 26 & 5 & 83.9 & \\
\hline 2005,2006 & 44 & 37 & 7 & 84.1 & \\
\hline
\end{tabular}

${ }^{\mathrm{a}}$ Fisher's exact test; ${ }^{\mathrm{b}} \chi^{2}$ test; NAC, neoadjuvant chemotherapy; BMI, body mass index.

Data analysis. Differences in changes in lymph-flow, identification rate of SLNs and changes in the number of detected SLNs were examined before and after NAC. Moreover, factors which might affect the detection of SLNs, such as $\mathrm{T}$ factors, $\mathrm{N}$ factors, patient age, BMI and primary tumor site, were compared with the identification rate of SLNs.

Differences in categoric variables were analyzed using the Chi-square test or Fisher's exact test. P values $<0.05$ were considered statistically significant. Dr. SPSS software for Windows was used throughout this study.

\section{Results}

The overall identification rate for SLNs using CT lymphography was $84 \%(63 / 75)$ (Table II). The identification rate was $79.5 \%$ (35/44) for examinations performed before NAC and/or for patients treated without chemotherapy and was $90.3 \%$ (28/31) for examinations performed just after NAC. Therefore, the identification rate following NAC tended to be higher than before NAC, although there is no statistically significant difference between the two.

The factor that most affected the identification rate of the SLNs was the existence of metastatic axillary lymph nodes.
The identification rate of the SLNs significantly decreased to $42.9 \%(6 / 14)$ for patients with positive-axillary lymph nodes. Therefore, SLNs could not be detected for 8 of the 12 patients with positive nodes. The identification rate of the SLNs was also lower for patients with primary tumors $\geq 2 \mathrm{~cm}$ in the longest diameter evaluated by MRI. Patient age, BMI, and affected regions of the breast showed no correlation with the identification rate of the SLNs.

As shown in Table III, the identification rate of the SLNs was also higher after NAC for patients who underwent CT lymphography both before and after NAC.

As shown in Table IV, the number of detected SLNs was higher in 6 patients after NAC compared with before NAC and was unchanged in 16 patients (Fig. 2). No patient showed a lower number of detected SLNs following NAC. Therefore, lymph-flow interruption due to NAC does not occur during the NAC treatment of 2 months.

For 2 patients with positive axillary nodes whose SLNs were not detected before chemotherapy, the SLNs became detectable after NAC. For 1 of these 2 patients the metastatic axillary lymph nodes were remarkably decreased in size by $\mathrm{NAC}$, and the disappearance of the metastatic lesions was confirmed by a histopathological examination of the surgically resected specimen (Fig. 3). 
Table III. Identification rate and number of detected SLNs before and after NAC.

\begin{tabular}{lrr}
\hline & Before NAC & After NAC \\
\hline Identification rate (\%) & 77.3 & 95.5 \\
Number of detected SLNs & $1.14 \pm 0.83$ & $1.45 \pm 0.67$ \\
(mean \pm SD) & & \\
\hline
\end{tabular}

SLNs, sentinel lymph nodes; NAC, neoadjuvant chemotherapy.
Table IV. Changes in the number of detected SLNs before and after NAC.

Number of detected SLNs (before $\rightarrow$ after NAC)

\begin{tabular}{lr}
\hline Increase & 6 cases \\
No change & 16 cases \\
Decrease & 0 cases
\end{tabular}

SLNs, sentinel lymph nodes; NAC, neoadjuvant chemotherapy.

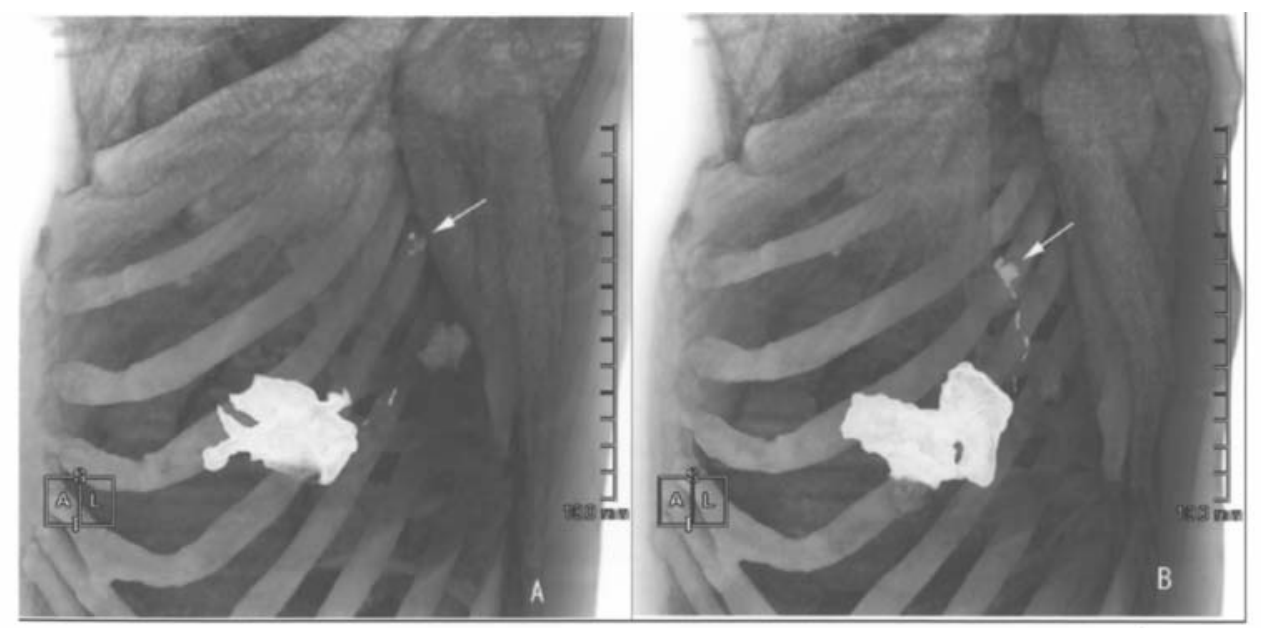

Figure 2. (A) Before neoadjuvant chemotherapy (NAC). The detection of sentinel lymph nodes (SLNs) $(\rightarrow$ ) is possible, but the contrast medium inflow is insufficient. (B) After NAC. The lymph-flow is clear and the detection of SLNs $(\rightarrow)$ is also easy. The existence of potential lymph node metastasis at the time of pre-NAC is suspected.

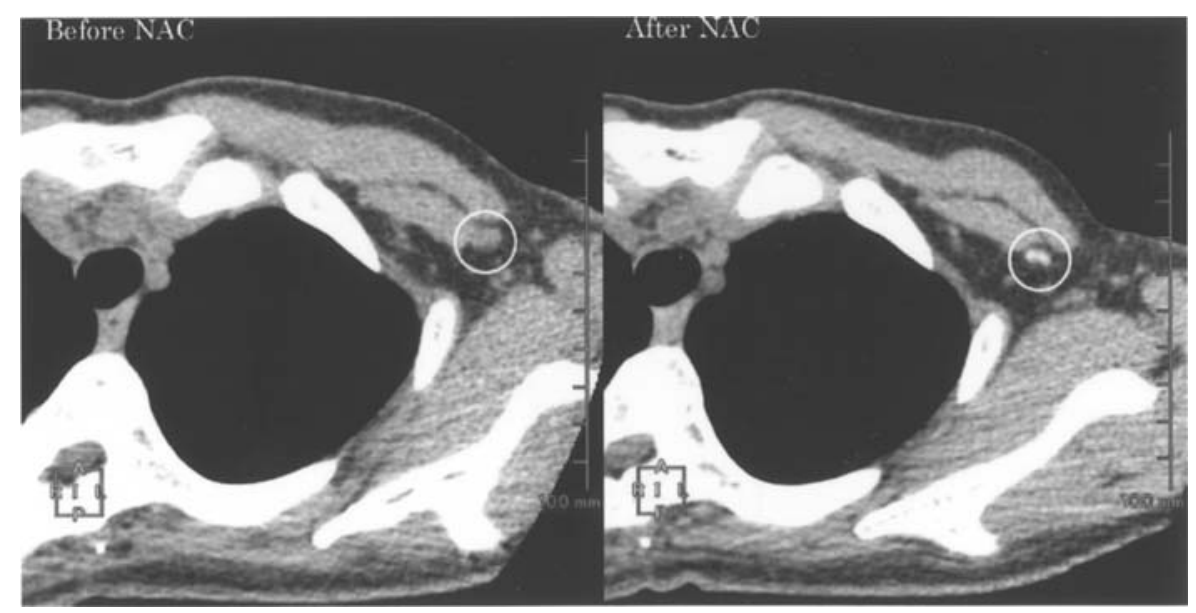

Figure 3. In computed tomography (CT) lymphography before neoadjuvant chemotherapy (NAC), the lymph-flow could not be observed and the sentinel lymph nodes (SLNs) were undetected. Due to NAC, the short diameter of the lymph node (o) was reduced to $3 \mathrm{~mm}$ from $7 \mathrm{~mm}$. CT lymphography following NAC detected an SLN, which could not be detected before NAC. A total glandectomy and axillary lymph node dissection were performed, histopathologically confirming that lymph node metastasis disappeared.

Almost all SLNs were detected in the axillary region of level I. In the patients who had SLNs in the axillary region of level II or the ipsilateral mammary gland (Fig. 4), CT lymphography was useful for identifying sentinel lymph nodes and for determining the axillary incision site.

\section{Discussion}

Factors which can affect the detectability of SLNs in patients with breast cancer are considered to be the technique used in the procedure, translocation of the contrast agent from the 

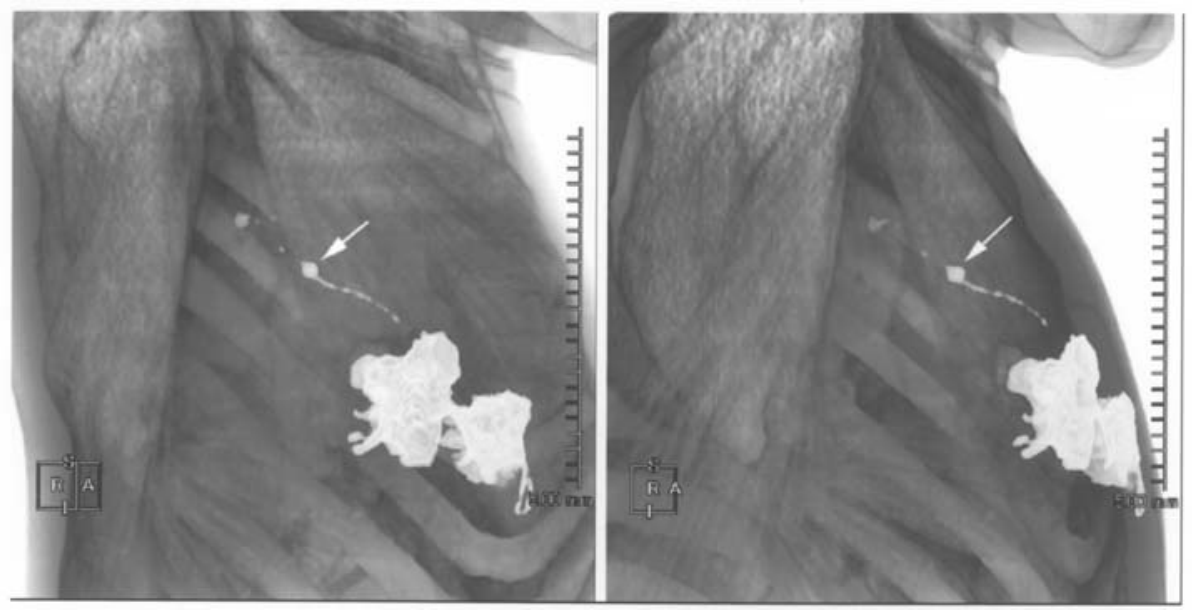

Figure 4. Inflow into the intramammary lymph node $\rightarrow$ ) was observed before the contrast medium flowed into the axillary lymph node, and it was considered as a sentinel lymph node (SLN). If computed tomography lymphography is not performed, an axillary lymph node could be extracted as a SLN

stroma to the lymphatic tissue, and drainage of the contrast agent into the lymphatic duct and lymph nodes. The problems associated with the techniques used for SLN detection include operator competency and the fact that some SLNs are difficult to detect in elderly or obese patients whose lymphatic tissues in the stroma are of relatively low density $(19,20)$. The identification rate of SLNs reflects the investigator's learning curve with procedures such as the dye method and RI method. At any given institution, the identification rate improves over time, with a corresponding decrease in the false-negative rate (21-23).

In terms of the influx of the contrast agent from the lymphatic duct and lymph nodes, we have to consider the factors of possible influx interruption, which could be caused by fibrotic changes induced by NAC and/or lymph node metastasis (24). In the present study, there was no interruption to the lymphatic influx and the identification rate of the SLNs was not decreased by NAC.

In our study, the factor that most affected the identification rate of the SLNs was the existence of metastasispositive lymph nodes, and the improvement of lymphatic drainage into those positive nodes was recognized following $\mathrm{NAC}$ in some cases. Among these cases, the lymph nodes decreased in size following NAC, leading to the conclusion that the interrupted drainage into the positive lymph nodes was improved by a size decrease of the metastatic lesion in the positive lymph nodes due to NAC.

Moreover, freshly detected SLNs following NAC, which had not been detected by CT lymphography performed before NAC, should be recognized clinically as metastasispositive lymph nodes. Therefore, for patients showing these findings by CT lymphography, SLNB could be contraindicated. Additionally, if we perform SLNB before NAC for these patients, lymph nodes other than true SLNs could be resected as false SLNs, and this is one of the factors giving rise to false-negatives in SLNB.

In the present study, there was no correlation between the identification rate and patient age or BMI. Nevertheless, we still believe these parameters to be identification rate-lowering factors in the use of CT lymphography as well as the dye method and RI method.
The T-factor of primary tumors also affected the identification rate of the SLNs in our study, most likely because the frequency of metastasis-positive lymph nodes depends on tumor size (25). The tumor growth does not affect the identification rate of the SLNs $(26,27)$. However, the accuracy of the SLN detection is reduced by the tumor growth, possibly due to the appearance of new routes of lymphatic influx from the primary tumor (28). Therefore, in patients with relatively large tumors, it is possible that the rate of false negative lymph nodes increases by performing SLNB. Thus, the size of the primary tumor at the initial examination could be an important factor in terms of the application of SLNB after NAC.

Patients who have clinically negative axillary lymph nodes and relatively small primary tumors before NAC are presumably good candidates for SLNB, and are also considered good candidates for the procedures following NAC. In the present study, there were few false-negative cases among these patients, validating the methods used.

The overall identification rate of the SLNs using CT lymphography was $84 \%$ (63/75), which was not altogether satisfactory. The patients with T1, T2-primary tumors and clinically negative axillary nodes showed a much higher SLN identification rate of $95.7 \%$ (22/23). Therefore, we concluded that we can reliably perform CT lymphography following NAC for these patients.

Another reason to hesitate before performing SLNB after NAC is the non-homogeneity of the neoadjuvant chemotherapeutic effect for each node in patients with multiple metastasis-positive lymph nodes. If a metastatic lesion in the sentinel lymph nodes disappears by NAC, the patient produces a false-negative result for the existence of residual metastatic lesions in the non-SLNs. To resolve this problem, CT lymphography is considered a valuable tool in terms of recognizing the sizes of both SLNs and non-SLNs. As the size recognition of the each lymph node using CT has been reported to be meaningful in distinguishing between metastasis-positive nodes and negative nodes $(29,30)$, we can also distinguish them based mainly on the decrease in size following NAC. Lymph nodes showing a relative decrease following chemotherapy are reliably considered as clinically- 
positive nodes at the time of pre-chemotherapy. Moreover, patients with smaller nodes following chemotherapy should be excluded from SLNB after NAC. Then, we will be able to achieve a decrease in the number of false-negative cases following NAC.

CT lymphography is technically easy to perform and the acquisition time is short, as there is no difference in the results at the time of the dividing period into the first and the second halves. In addition, CT lymphography can be performed at any institution equipped with a multi-row detector CT. This method also allows for the detection of the site of the SLNs pre-operatively. Thus, the incision site on the axillary region can be easily determined. It is especially valuable to identify the lymphatic drainage and the site of SLNs for use in the dye method, which cannot identify these features.

The present results indicate diagnosis can be improved by comparing changes in the lymph-flow or SLNs by CT lymphography before and after NAC. Therefore, it is possible that false negative cases could be lessened, choosing the safer option of SLNB after NAC. For breast cancer patients who desire SLNB instead of ALND after NAC, it is very useful to perform CT lymphography before and after NAC due to its efficacy in indicating whether SLNB should be performed after NAC.

\section{References}

1. Fisher B, Jeong JH, Anderson S, et al: Twenty-five-year followup of a randomized trial comparing radical mastectomy, total mastectomy, and total mastectomy followed by irradiation. $\mathrm{N}$ Engl J Med 347: 567-575, 2002.

2. Haffty BG, Ward B, Pathare P, et al: Reappraisal of the role of axillary lymph node dissection in the conservative treatment of breast cancer. J Clin Oncol 15: 691-700, 1997.

3. Hoebers FJ, Borger JH, Hart AA, et al: Primary axillary radiotherapy as axillary treatment in breast-conserving therapy for patients with breast carcinoma and clinically negative axillary lymph nodes. Cancer 88: 1633-1642, 2000.

4. Veronesi U, Paganelli G, Viale G, et al: A randomized comparison of sentinel-node biopsy with routine axillary dissection in breast cancer. N Engl J Med 349: 546-553, 2003.

5. Tanaka Y, Maeda H, Ogawa Y, et al: Sentinel node biopsy in breast cancer patients treated with neoadjuvant chemotherapy. Oncol Rep 15: 927-931, 2006.

6. Shimazu K, Tamaki Y, Taguchi T, et al: Sentinel lymph node biopsy using periareolar injection of radiocolloid for patients with neoadjuvant chemotherapy-treated breast carcinoma. Cancer 100: 2555-2561, 2004.

7. Breslin TM, Cohen L, Sahin A, et al: Sentinel lymph node biopsy is accurate after neoadjuvant chemotherapy for breast cancer. J Clin Oncol 18: 3480-3486, 2000.

8. Kuerer HM, Sahin AA, Hunt KK, et al: Incidence and impact of documented eradication of breast cancer axillary lymph node metastases before surgery in patients treated with neoadjuvant chemotherapy. Ann Surg 230: 72-78, 1999.

9. Fisher ER, Wang J, Bryant J, et al: Pathobiology of preoperative chemotherapy: findings from the National Surgical Adjuvant Breast and Bowel (NSABP) protocol B-18. Cancer 95: 681-695, 2002.

10. Honkoop AH, Pinedo HM, De Jong JS, et al: Effects of chemotherapy on pathologic and biologic characteristics of locally advanced breast cancer. Am J Clin Pathol 107: 211-218, 1997.
11. Mamounas EP, Brown A, Anderson S, et al: Sentinel node biopsy after neoadjuvant chemotherapy in breast cancer: results from National Surgical Adjuvant Breast and Bowel Project Protocol B-27. J Clin Oncol 23: 2694-2702, 2005.

12. Schrenk P, Hochreiner G, Fridrik M, et al: Sentinel node biopsy performed before preoperative chemotherapy for axillary lymph node staging in breast cancer. Breast J 9(4):282-287, 2003.

13. Pendas S, Jakub J, Giuliano R et al: The role of sentinel lymph node biopsy in patients with ductal carcinoma in situ or with locally advanced breast cancer receiving neoadjuvant chemotherapy. Cancer Control 11: 231-235, 2004.

14. Trocha SD and Giuliano AE: Sentinel node in the era of neoadjuvant therapy and locally advanced breast cancer (Review). Surg Oncol 12: 271-276, 2003.

15. Suga K, Yuan Y, Okada M, et al: Breast sentinel lymph node mapping at CT lymphography with iopamidol: preliminary experience. Radiology 230: 543-552, 2004.

16. Minato M, Hirose C, Sasa M, et al: 3-dimensional computed tomography lymphography-guided identification of sentinel lymph nodes in breast cancer patients using subcutaneous injection of nonionic contrast medium: a clinical trial. J Comput Assist Tomogr 28: 46-51, 2004.

17. Tangoku A, Yamamoto S, Suga K, et al: Sentinel lymph node biopsy using computed tomography-lymphography in patients with breast cancer. Surgery 135: 258-265, 2004.

18. Ogawa Y, Nishioka A, Inomata $\mathrm{T}$, et al: Conservation treatment intensified with an anti-estrogen agent and CAF chemotherapy for stage I and II breast cancer. Oncol Rep 7: 479-484, 2000.

19. Krag D, Weaver D, Ashikaga T, et al: The sentinel node in breast cancer - a multicenter validation study. N Engl J Med 339: 941-946, 1998.

20. Sener SF, Winchester DJ, Brinkmann E, et al: Failure of sentinel lymph node mapping in patients with breast cancer. J Am Coll Surg 198: 732-736, 2004.

21. Cox CE, Pendas S, Cox JM, et al: Guidelines for sentinel node biopsy and lymphatic mapping of patients with breast cancer. Ann Surg 227: 645-653, 1998.

22. Johnson JM, Orr RK and Moline SR: Institutional learning curve for sentinel node biopsy at a community teaching hospital. Am Surg 67: 1030-1033, 2001.

23. Classe JM, Curtet C, Campion L, et al: Learning curve for the detection of axillary sentinel lymph node in breast cancer. Eur J Surg Oncol 29: 426-433, 2003.

24. Brenot-Rossi I, Houvenaeghel G, Jacquemier J, et al: Nonvisualization of axillary sentinel node during lymphoscintigraphy: is there a pathologic significance in breast cancer? J Nucl Med 44: 1232-1237, 2003.

25. Silverstein MJ, Skinner KA and Lomis TJ: Predicting axillary nodal positivity in 2282 patients with breast carcinoma. World J Surg 25: 767-772, 2001.

26. Bedrosian I, Reynolds C, Mick R, et al: Accuracy of sentinel lymph node biopsy in patients with large primary breast tumors. Cancer 88: 2540-2545, 2000.

27. McMasters KM, Tuttle TM, Carlson DJ, et al: Sentinel lymph node biopsy for breast cancer: a suitable alternative to routine axillary dissection in multi-institutional practice when optimal technique is used. J Clin Oncol 18: 2560-2566, 2000.

28. Noguchi M, Motomura K, Imoto S, et al: A multicenter validation study of sentinel lymph node biopsy by the Japanese Breast Cancer Society. Breast Cancer Res Treat 63: 31-40, 2000.

29. Hata Y, Ogawa Y, Nishioka A, et al: Thin section computed tomography in the prone position for detection of axillary lymph node metastases in breast cancer. Oncol Rep 5: 1403-1406, 1998.

30. Ogawa Y, Nishioka A, Nishigawa T, et al: Thin-section CT evaluation and pathologic correlation of therapeutic effect of neoadjuvant chemotherapy for axillary lymph nodes of clinically node-positive breast cancer patients. Oncol Rep 10: 985-989, 2003. 